\title{
Different Expressions and DNA Methylation Patterns of Lysophosphatidic Acid Receptor Genes in Mouse Tumor Cells
}

\author{
Kyoko Okabe ${ }^{a}$ Mai Hayashi ${ }^{a}$ Naoko Wakabayashia Yasuna Yamawaki ${ }^{a}$ \\ Miki Teranishi $^{a}$ Nobuyuki Fukushima ${ }^{b}$ Toshifumi Tsujiuchi ${ }^{a}$ \\ Divisions of a Cancer Biology and Bioinformatics and ${ }^{b}$ Molecular Neurobiology, Department of Life Science, \\ Faculty of Science and Technology, Kinki University, Higashiosaka, Japan
}

\section{Key Words}

Mouse $\cdot$ Lysophosphatidic acid $\cdot$ Lysophosphatidic acid receptor $\cdot$ DNA methylation $\cdot$ Cancer cell

\begin{abstract}
Objective: Lysophosphatidic acid (LPA) receptors act as several biological effectors through LPA, which is a bioactive phospholipid. Recently, aberrant expressions of LPA receptor genes due to DNA methylation have been detected in several tumor cells. In this study, we measured expression levels and DNA methylation status of LPA receptor genes in mouse tumor cells, LL/2 lung carcinoma, B16F0 melanoma, FM3A mammary carcinoma and L1210 leukemia cells, compared with normal tissues. Methods: Total RNAs were extracted and RT-PCR analysis was performed. For DNA methylation status, bisulfite sequencing analysis was carried out, comparing outcomes with other tumor cells and normal tissues. Results: The expressions of LPA1 gene were shown in $\mathrm{LL} / 2$, but not in B16F0, FM3A and L1210 cells. While the LPA2 gene was expressed in all 4 tumor cells, the LPA3 gene was unexpressed in them. The LPA1 and LPA3 unexpressed cells were highly methylated, although normal tissues were all unmethylated. The DNA methylation status was correlated
\end{abstract}

with gene expression levels in cancer cells. Conclusion: The present results demonstrate that DNA methylation patterns of LPA receptor genes are dependent on cancer cell types, suggesting that LPA receptors may be new molecular targets for therapeutic approaches and chemoprevention.

Copyright $\odot 2011$ S. Karger AG, Basel

\section{Introduction}

Lysophosphatidic acid (LPA) is a bioactive mediator and has several cellular effects, such as cell proliferation, differentiation, migration, morphogenesis and protection from apoptosis $[1,2]$. In human cancer cells, LPA can enhance not only cell proliferative activity, but also metastatic potency, including cell migration, invasion and production of angiogenic factors [3-6].

LPA interacts with LPA receptors which are $G$ proteincoupled transmembrane receptors $[1,2]$. So far, 6 LPA receptors have been identified, LPA1, LPA2, LPA3, LPA4, LPA5 and LPA6 [1, 2, 7-13]. These receptors show different expression patterns in normal tissues and biological responses to LPA [1, 2]. Previously, aberrant expressions of LPA receptors have been found in human several tu-

\section{KARGER}

Fax +4161306 1234

E-Mail karger@karger.ch

www.karger.com
(C) 2011 S. Karger AG, Basel

1015-2008/10/0776-0309\$26.00/0

Accessible online at:

www.karger.com/pat
Dr. Toshifumi Tsujiuchi

Division of Cancer Biology and Bioinformatics, Department of Life Science Faculty of Science and Engineering, Kinki University, 3-4-1, Kowakae Higashiosaka, Osaka 577-8502 (Japan)

Tel. +81 66772 2332, Fax +81 66723 2721, E-Mail ttujiuch@life.kindai.ac.jp 
mors, demonstrating that LPA receptors might be involved in the acquisition of the growth advantage of tumor cells $[1-6,14,15]$. Our recent reports have indicated that distinct expression patterns of LPA receptor genes occur in human colon cancer cells [16]. In rodents, loss of LPA1 expression due to its aberrant DNA methylation was shown in rat tumor cells [17]. On the other hand, mutations of LPA receptor genes have also been detected in human and rodents tumors $[16,18,19]$. Therefore, it is suggested that alterations of LPA receptor genes may play important roles in the pathogenesis of tumor cells as well as LPA per se.

So far, alterations of LPA1, LPA2 and LPA3 receptors have been relatively well investigated in tumor cells, but not those of other receptors $[1-6,14,15]$. In this study, we measured the expression levels of LPA1, LPA2 and LPA3 genes and their DNA methylation status in mouse tumor cells, LL/2 lung carcinoma, B16F0 melanoma, FM3A mammary carcinoma and L1210 leukemia cells. Moreover, we also investigated the effects of LPA on cell proliferation of mouse tumor cells. The present results indicate that expression patterns and DNA methylation status of LPA receptor genes are distinct in mouse tumor cells, demonstrating that with the investigations for LPA receptor genes used these tumor cells would be informative and valuable for developing novel therapeutic and chemopreventive agents.

\section{Materials and Methods}

\section{Cell Culture and Animals}

The 4 mouse tumor cell lines used in this study were LL/2 lung carcinoma, B16F0 melanoma, FM3A mammary carcinoma and L1210 leukemia cells. All cell lines were cultured in DMEM containing $10 \%$ fetal bovine serum in $5 \% \mathrm{CO}_{2}$ atmosphere at $37^{\circ} \mathrm{C}$. In order to obtain normal lung, skin, mammary gland tissues and blood, ICR female mice (Japan SLC Inc., Shizuoka, Japan) was used.

\section{Semi-Quantitative RT-PCR Amplification for the LPA1 and} LPA3 Gene Expressions

Total RNA was extracted from each cell and tissue using ISOGEN (Nippon Gene Inc., Toyama, Japan) and first-strand cDNA was synthesized from $0.5-\mu \mathrm{g}$ samples with Transcriptor First-Strand cDNA Synthesis Kit (Roche Diagnostics Co. Ltd., Mannheim, Germany).

Semi-quantitative RT-PCR analysis was performed as described previously $[16,17]$. Primer pairs used in this study were as follows: for mouse LPA1 (NCBI accession No. NC_000070), F: 5'-TCCCCTCAACCACACCATTCT-3', R: 5'-GGCTGGCTTCCTTCTAAACC-3' (annealing temperature: $64^{\circ} \mathrm{C}$ ); for mouse LPA2 (NCBI accession No. NC_000074), F: 5'-AAAGGCTGGTTCCTGCGACA-3'， R: 5'-TGCTCTGCCATGCGTTCAAC-3' (annealing temperature: $62^{\circ} \mathrm{C}$ ); for mouse LPA3 (NCBI accessionNo. NC_000069), F: 5'-TTGCTGGTTATTGCTGTGGA-3', R: 5'-CGCTTTTTGGTCAAGTTGCT-3' (annealing temperature: $64^{\circ} \mathrm{C}$ ). The mouse glyceraldehyde-3-phosphate dehydrogenase gene was used as an internal control gene. For each gene, multiple cycles of PCR amplification were tested and the most appropriate cycle number was determined. The amplified products were then separated on $2 \%$ agarose gels containing $0.05 \mu \mathrm{g} / \mathrm{ml}$ ethidium bromide $[16,17]$.

\section{Bisulfite Sequencing Analysis}

Genomic DNA was extracted with a DNeasy tissue kit (Qiagen, Hilden, Germany) from each cell and tissue, and treated with an EpiTect Bisulfite Kit (Qiagen). For bisulfite sequencing, PCR was performed with the following primer sets: LPA1 (NCBI accession No. NP_034466.1), BS-F 5'-TTATTAAAGTTTTGTAAGAGGTTTGG-3', BS-R 5'-CACAAAACACAAATCAAAAACTCCC-3' (annealing temperature: $60^{\circ} \mathrm{C}$ ); LPA3 (NCBI accession No. NM_022983.3), BS-F 5'-GGGGATTTTAGGTAGTTTTTTAGAG-3', BS-R 5'-AAAATCCCCAACAACTTTACTAAAAC-3' (annealing temperature: $57^{\circ} \mathrm{C}$ ). PCR products were subcloned with a DynaExpress TA PCR cloning kit (BioDynamics Laboratory Inc., Tokyo, Japan) and sequenced with a BigDye terminator v3.0 cycle sequencing ready reaction kit (Applied Biosystems, Tokyo, Japan) and an ABI PRISM 310 genetic analyzer (Applied Biosystems). For each sample, 8 clones were sequenced [16, 17].

\section{Cell Proliferation Assay}

Cells were plated at 2,000 cells/well in a 96-well plate and cultured for 1 day. Cells were washed with serum-free DMEM and further cultured with $100 \mu \mathrm{l}$ of serum-free DMEM. Cells were treated with 1 or $10 \mu \mathrm{M}$ LPA (Avanti Polar Lipids Inc., Alabaster, Ala., USA) for 3 days, which were added every $24 \mathrm{~h}$. For cell proliferation assay, solution from a cell counting kit 8 (CCK-8; Dojin Chemistry, Kumamoto, Japan) was added to the plate and cells were further incubated for $1 \mathrm{~h}$. The absorbance of the culture medium at $450 \mathrm{~nm}$ was determined. The assay was always done in triplicate [20].

\section{Results}

The expression levels of LPA1, LPA2 and LPA3 genes in mouse tumor cells were measured by semi-quantitative RT-PCR analysis, comparing them with normal tissues of lung, skin, mammary gland and blood. Representative results are shown in figure 1 . The expressions of LPA1 gene were shown in LL/2, but unexpressed in B16F0, FM3A and L1210 cells. Although the LPA2 gene was expressed in all tumor cells, the LPA3 gene unexpressed in them (fig. 1a). Since LL/2, B16F0, FM3A and L1210 cells were derived from lung, skin, mammary gland and blood tissues, respectively, we also assessed expression levels in those tissues. Normal lung, skin, mammary gland and blood tissues expressed LPA1, LPA2 and LPA3 genes (fig. 1b). 
Fig. 1. Expressions of LPA receptor genes by semi-quantitative RT-PCR analysis.
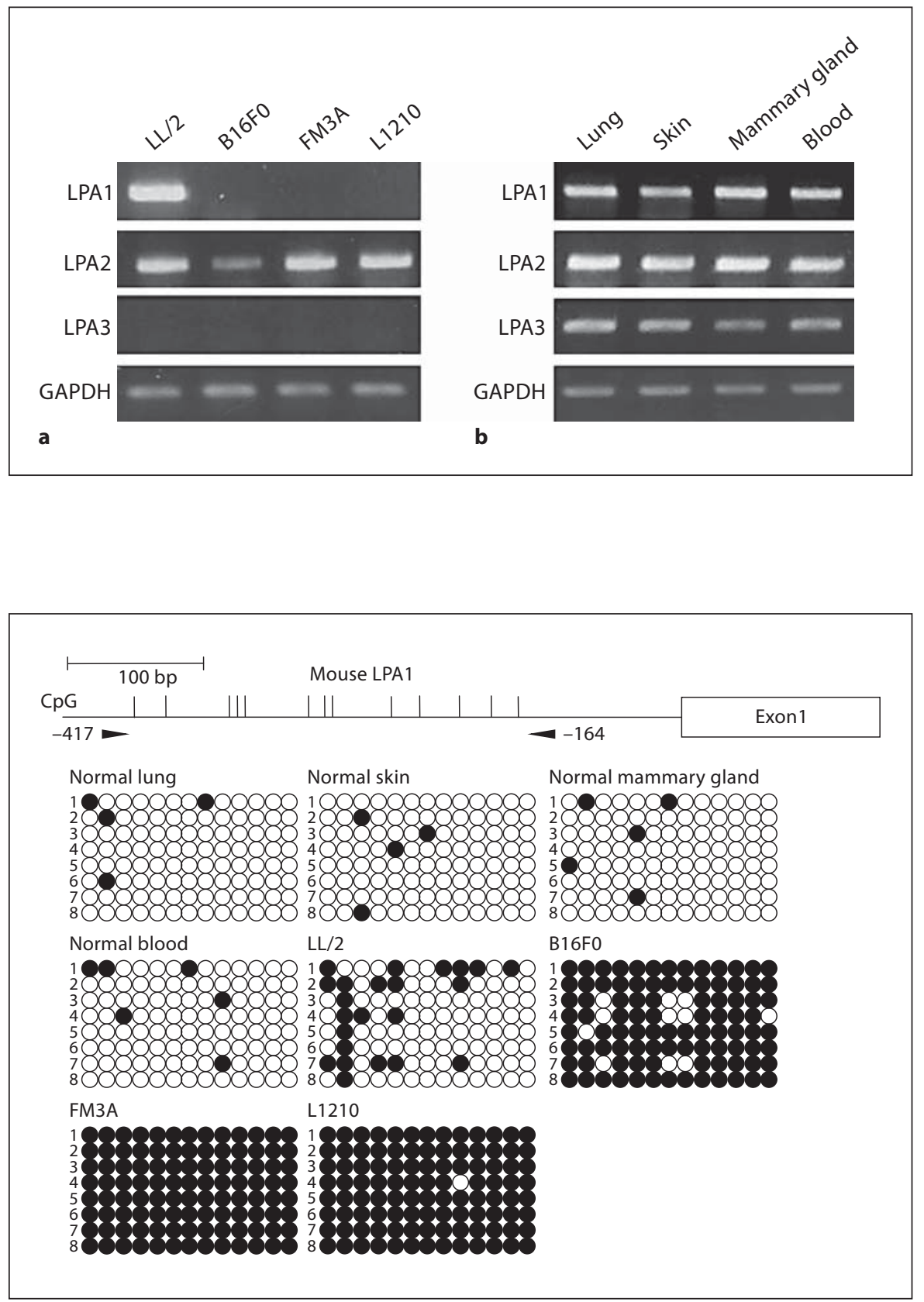

Fig. 2. Results of the methylation analysis of the $5^{\prime}$ upstream region of the mouse LPA1 gene evaluated by bisulfite sequencing. For each sample, 8 clones were sequenced. The primer pair used for the bisulfite sequencing is indicated by arrow heads. Methylated CpG sites are shown by closed circles and unmethylated CpG sites are shown by open circles. The first base of exon 1 was defined +1 .
Since loss of expressions was found in LPA1 and LPA3 genes, we next performed a bisulfite sequence analysis to measure methylation status of the $5^{\prime}$ upstream region of LPA1 (between nt. -417 and $-164 ; 13$ CpG sites) and LPA3 (between nt. -213 and $-5 ; 17$ CpG sites) in tumor cells. LPA1 gene was weakly methylated in LL/2 cells. By contrast, B16F0, FM3A and L1210 cells were highly methylated (fig. 2). LPA3 gene was highly methylated in all tumor cells (fig. 3). Normal lung, skin, mammary gland and blood tissues were unmethylated in LPA1 and LPA3 genes (fig. 2, 3). The DNA methylation status of LPA 1 and LPA 3 genes was correlated with expression levels.

We also examined the effects of LPA on cell proliferation of mouse tumor cells. LPA showed significant growth stimulation at $1 \mu \mathrm{M}$ and $10 \mu \mathrm{M}$ in LL/2 and B16F0 cells 
Fig. 3. Results of the methylation analysis of the $5^{\prime}$ upstream region of the mouse LPA3 gene evaluated by bisulfite sequencing. For each sample, 8 clones were sequenced. The primer pair used for the bisulfite sequencing is indicated by arrow heads. Methylated CpG sites are shown by closed circles and unmethylated $\mathrm{CpG}$ sites are shown by open circles. The first base of exon 1 was defined +1 .

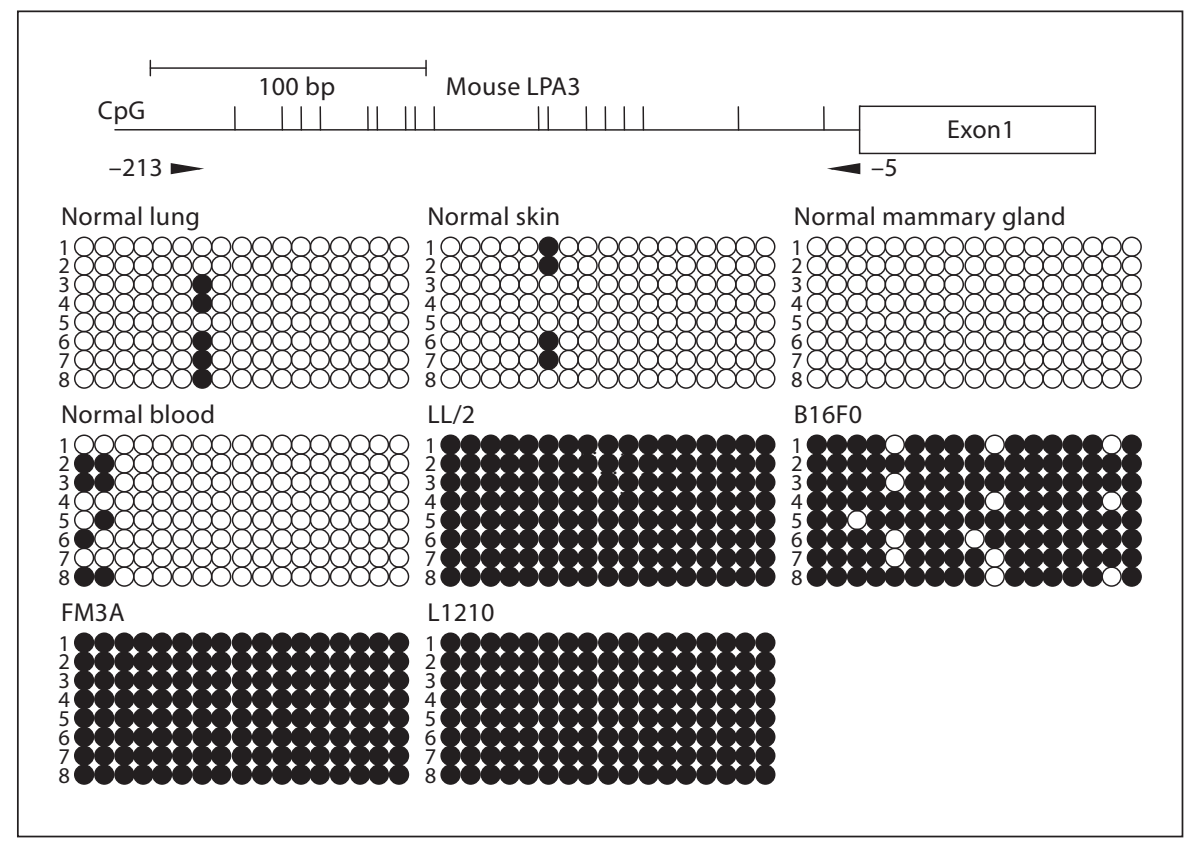

$(\mathrm{p}<0.05$ and $\mathrm{p}<0.001$, respectively), and at $10 \mu \mathrm{M}$ in FM3A cells ( $\mathrm{p}<0.001)$. By contrast, no effect of LPA on cell growth was found in L1210 cells (fig. 4).

\section{Discussion}

The expression patterns of LPA receptor genes in normal tissues were various $[1,2]$. LPA 1 is ubiquitously expressed in normal tissues and the expressions of LPA2 and LPA 3 are relatively restricted, suggesting that these receptors have different biological functions $[1,2]$. In human ovarian cancer cells, the expression levels of LPA2 and LPA3 genes in ovarian cancer cells were markedly higher than those in normal ovarian epithelial cells, but LPA1 gene expression levels were various [3-5]. By contrast, while the expression levels of LPA1 and LPA3 genes were relatively low, the LPA2 gene was overexpressed in human colon cancer tissues [15]. Recently, we have shown various levels of LPA1 and LPA3 expressions in human colon cancer cells, but well-expressed LPA2, correlating with DNA methylation status [16]. These findings suggest that alterations of LPA receptors may be involved in the acquisition of the growth advantage of tumor cells in human carcinogenesis. The present study indicated that different expression levels of LPA receptor genes due to DNA methylation were also found in mouse tumor cells. There-

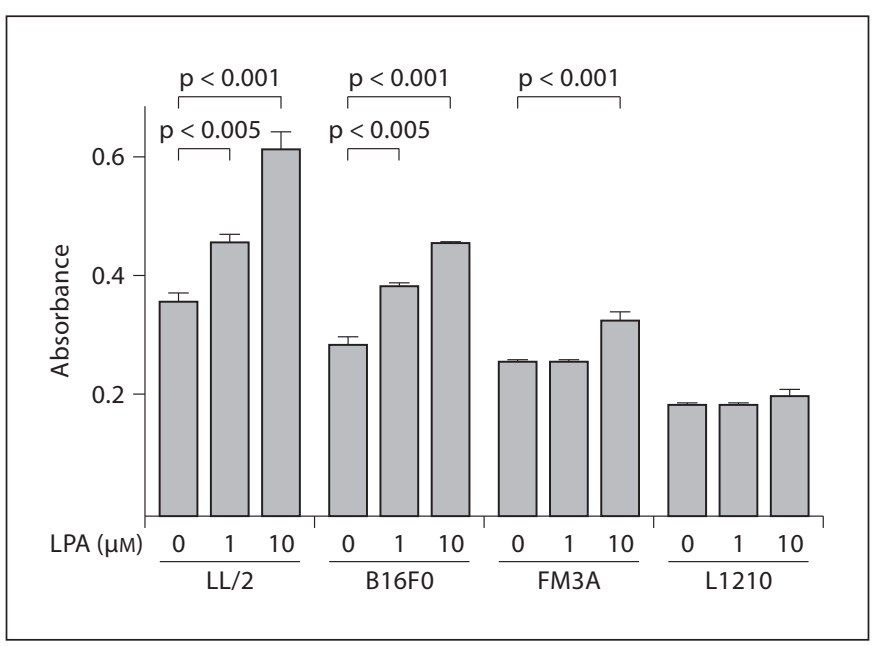

Fig. 4. The effects of LPA on cell growth in mouse tumor cells. Cells were cultured with or without LPA $(1$ or $10 \mu \mathrm{M})$, and cell proliferation was measured using a CCK-8. Columns indicate the mean of 3 studies performed in triplicate; bars indicate SD.

fore, it is suggested that DNA methylation status of LPA receptor genes may be dependent on tumor cell type.

It has been considered that LPA receptors may have different responses to LPA $[1,2]$. In fact, LPA could induce a wide range of cellular responses throughout LPA1 or 
LPA2, such as cell proliferation, phospholipase $\mathrm{C}$ activation, intracellular calcium mobilization and adenylyl cyclase inhibition $[1,2]$. In early pregnancy, LPA3 had a critical function in spacing and implantation of embryos [21, 22]. Moreover, different responses of LPA receptors to LPA have also been reported in human cancer cells $[3,5$, $6,14]$. The ovarian cancer cells with high expression of LPA1 indicated lower cell proliferation, cell death through apoptosis and anoikis to LPA, suggesting that LPA1 may act as a negative regulator for cell growth $[3,5,6]$. By contrast, LPA stimulated cell migration, proliferation and adhesion in the LPA1-expressing colon cancer cells [14]. In the present study, we indicated the different effects of LPA on cell proliferaion in mouse tumor cells. Therefore, it is suggested that the different expression pattern of LPA receptor genes may be related to clinically characteristic features of cancer cells through the distinct responses to LPA $[14,15]$.

Previously, we have reported that frequent mutations of the LPA1 gene were not only detected in rat lung adenocarcinomas but also in preneoplastic lesions [19]. Moreover, our recent studies have indicated that LPA2 and LPA4 gene mutations occurred in human colon cancer cells [16], and LPA1 and LPA3 gene mutations in human sarcoma cells [18]. These findings suggest that LPA receptor gene mutations may play an important role in the pathogenesis of tumor cells as well as aberrant expressions due to DNA methylation. In the mouse tumor cells used in this study, we could not detect LPA receptor gene mutations (data not shown).

In conclusion, we demonstrate that different expressions and DNA methylation patterns of LPA receptor genes are found in mouse tumor cells, suggesting that LPA receptor gene expressions may be dependent on tumor cell type, which has possible implications for new therapeutic and chemoprevention approaches. To better understand the biological significance of LPA receptors in tumor cells, we are currently investigating functional analyses for LPA receptors in several cancer cells with different expression levels of LPA receptor genes.

\section{Acknowledgments}

This study was supported in part by the Foundation for Promotion of Cancer Research in Japan, a Grant-in-Aid (20591765) for Scientific Research from the Ministry of Education, Culture, Sports, Science and Technology of Japan, grants (21321201) from the Ministry of Health, Labor and Welfare of Japan, and by grants (RK-027) from the Faculty of Science and Engineering, Kinki University, Osaka, Japan.

\section{References}

1 Contos JJA, Ishii I, Chun J: Lysophosphatidic acid receptors. Mol Pharmacol 2000;58: $1188-1196$

- Ishii I, Fukushima N, Ye X, Chun J: Lysophospholipid receptors: signaling and biology. Annu Rev Biochem 2004;73:321-354.

-3 Furui T, LaPushin R, Mao M, Khan H, Watt SR, Watt MAV: Overexpression of edg-2/vzg1 induces apoptosis and anoikis in ovarian cancer cells in a lysophatidic acid-independent manner. Clinical Cancer Res 1999;5: 4308-4318.

4 Goetzl EJ, Dolezalova H, Kong Y, Hu YL, Jaffe RB, Kalli KR, Conover CA: Distinctive expression and functions of the type 4 endothelial differentiation gene-encoded G protein-coupled receptor for lysophosphatidic acid in ovarian cancer. Cancer Res 1999;59: 5370-5375.

5 Fang X, Schummer M, Mao M, Yu S, Tabassam FH, Swaby R, Hasegawa Y, Tanyi JL, LaPushin R, Eder A, Jaffe R, Erickson J, Mills GB: Lysophosphatidic acid is a bioactive mediator in ovarian cancer. Biochim Biophys Acta 2002;1582:257-264.

\footnotetext{
Fujita T, Miyamoto S, Onoyama I, Sonoda K, 10 Noguchi K, Ishii S, Shimizu T: Identification Mekada E, Nakano H: Expression of lysophosphatidic acid receptors and vascular endothelial growth factor mediating lysophosphatidic acid in the development of human ovarian cancer. Cancer Lett 2003;192:161169.

-7 Contos JJA, Ishii I, Fukushima N, Kingsbury MA, Ye X, Kawamura S, Brown JH, Chun J: Characterization of lpa(2) (Edg4) and lpa(1)/ lpa(2) (Edg2/Edg4) lysophosphatidic acid receptor knockout mice: signaling deficits without obvious phenotypic abnormality attributable to lpa(2). Mol Cell Bio 2002;22: 6921-6929.

-8 An S, Bleu T, Halmark OG, Goetzl EJ: Characterization of a novel subtype of human G protein-coupled receptor for lysophosphatidic acid. J Biol Chem 1998;273:7906-7910.

-9 Bandoh K, Aoki J, Hosono H, Kobayashi S, Kobayashi T, Murakami-Murofushi K, Tsujimoto M, Arai H, Inoue K: Molecular cloning and characterization of a novel human

\author{
of p2y9/GPR23 as a novel G protein-coupled \\ receptor for lysophosphatidic acid, structur- \\ ally distant from the Edg family. J Biol Chem \\ 2003;278:25600-25606. \\ Chun J: GPR92 as a new G12/13 and Gq cou- \\ pled lysophosphatidic acid receptor that in- \\ creases cAMP: LPA5. J Biol Chem 2006;281: \\ 23589-23597. \\ Petukhowa L, Gordon D, Christiano AM: \\ Disruption of P2RY5, an orphan G protein- \\ coupled receptor, underlies autosomal reces- \\ sive woolly hair. Nature Genet 2008;40:335- \\ 339. \\ Lee YA, Rüschendorf F, Voss K, Hillmer AM, \\ Molderings GJ, Franz T, Ramirez A, Nürn- \\ berg P, Nöthen MM, Berz RC: G protein-cou- \\ pled receptor P2Y5 and its ligand LPA are \\ involved in maintenance of human hair \\ growth. Nature Genet 2008;40:329-334.
}

$\rightarrow 11$ Lee CW, Rivera R, Gardell S, Dubin AE,

12 Shimomura Y, Wajid M, Ishii Y, Shapiro L,

13 Pasternack SM, von Kügelgen I, Aboud KAJ,
} G-protein-coupled receptor, EDG7, for lysophosphatidic acid. J Biol Chem 1999;274: 27776-27785. 
14 Shida D, Kitayama J, Yanaguchi H, Okaji Y, Tsuno NH, Watanabe T, Takuwa Y, Nagawa $\mathrm{H}$ : Lysophosphatidic acid (LPA) enhances the metastatic potential of human colon carcinoma DLD1 cells through LPA1. Cancer Res 2003;63:1706-1711.

15 Shida D, Watanabe T, Aoki J, Hama K, Kitayama J, Sonoda H, Kishi Y, Yamaguchi H, Sasaki S, Sako A, Konishi T, Arai H, Nagawa H: Aberrant expression of lysophosphatidic acid (LPA) receptors in human colorectal cancer. Lab Investigation 2004;84:13521362.

16 Tsujino M, Fujii M, Okabe K, Mori T, Fukushima N, Tsujiuchi T: Differential expressions and DNA methylation patterns of lysophosphatidic acid receptor genes in human colon cancer cells. Virchows Arch 2010; in press.
17 Tsujiuchi T, Shimizu K, Onishi M, Sugata E, Fujii H, Mori T, Honoki K, Fukushima N: Involvement of aberrant DNA methylation on reduced expression of lysophosphatidic acid receptor-1 gene in rat tumor cell lines. Biochem Biophys Res Commun 2006;349:11511155 .

18 Okabe K, Hayashi M, Fujii M, Honoki K, Mori T, Fukushima N, Tsujiuchi T: Mutations of lysophosphatidic acid receptor genes in human osteosarcoma cells. Pathobiology 2010;in press.

19 Yamada T, Furukawa M, Hotta M, Yamasaki A, Honoki K, Fukushima N, Tsujiuchi T: Mutations of lysophosphatidic acid receptor-1 gene during progression of lung tumors in rats. Biochem Biophys Res Commun 2009; 378:424-427.
20 Shano S, Hatanaka K, Ninose S, Moriyama R, Tsujiuchi T, Fukushima N: A Lysophosphatidic acid receptor lacking the PDZbinding domain is constitutively active and stimulates cell proliferation. Biochim Biophys Acta 2008; 1783:748-759.

-21 Ye X, Hama K, Contos JJ, Anliker B, Inoue A, Skinner MK, Suzuki H, Amano T, Kennedy G, Arai H, Aoki J, Chun J: LPA3-mediated lysoposphatidic acid signaling in embryo implantation and spacing. Nature 2005; 435 : 104-108.

22 Hama K, Aoki J, Inoue A, Endo T, Amano T, Motoki R, Kanai M, Ye X, Chun J, Matsuki N, Suzuki H, Shibasaki M, Arai H: Embryo spacing and implantation timing are differentially regulated by LPA3-mediated lysophosphatidic acid signaling in mice. Biol Reprod 2007;77:954-959. 